\title{
The Threshold for Favorable Outcome should be Set According to Functional Independence Measure Score at Admission in Logistic Regression Analysis
}

\author{
Makoto Tokunaga $^{1^{*}}$, Hirotaka Tanaka ${ }^{2}$, Naoshi Nishimura ${ }^{3}$, Katsunobu Sugihara ${ }^{4}$ and Hiroshi Kamoshita \\ ${ }^{1}$ Department of Rehabilitation, Kumamoto Kinoh Hospital, Kumamoto, Japan \\ ${ }^{2}$ Department of Rehabilitation, Chubu Rosai Hospital, Nagoya, Aichi, Japan \\ ${ }^{3}$ Department of Rehabilitation, Moriyama Memorial Hospital, Tokyo, Japan \\ ${ }^{4}$ Department of Rehabilitation, Hiroshima City Rehabilitation Hospital, Hiroshima, Japan
}

${ }^{5}$ Department of Rehabilitation, Tanashi Hospital, Tokyo, Japan

\begin{abstract}
Background: Logistic regression analysis setting Functional Independence Measure (FIM) score of 80 points or above at discharge as favorable rehabilitation outcome is reported. But this method is problematic because whether or not FIM score at discharge becomes greater than the fixed score depends on the FIM scores at admission. Therefore, we stratified FIM scores at admission and set thresholds in each stratified group.

Methods: In total, 290 patients with stroke hospitalized in a convalescent rehabilitation ward were included in this study. Their FIM scores at admission were all less than 80 points. Logistic regression analysis was performed on six explanatory variables including age, sex, type of stroke, number of days from onset to admission, motor FIM score at admission, and cognitive FIM score at admission. The objective variable was FIM at discharge ( 0 or 1$)$. We defined favorable outcome in two ways. In the conventional method, FIM scores at discharge of 80 points and above were defined as the favorable outcome. In the new method, FIM scores at admission were stratified into seven groups and the median value and above of FIM scores at discharge in each group were defined as the favorable outcome.

Results: FIM score at discharge was favorable if FIM scores at admission were higher in the conventional method, but independent from FIM scores at admission in the new method.

Conclusion: To investigate factors influencing FIM improvement using logistic regression analysis, the threshold for favorable outcome should be set according to FIM scores at admission.
\end{abstract}

\section{Introduction}

Multiple regression analysis is used when predicting objective variable using multiple explanatory variables. It is also used to find out how much influence the factor (explanatory variable) has on the outcome (objective variable). There are many reports of multiple regression analysis predicting Functional Independence Measure (FIM) scores at discharge or FIM gain (FIM score at discharge - FIM score at admission) [1-3]. But since multiple regression analysis is a parametric method, both the objective variable and explanatory variables are required for normal distribution.

On the other hand, logistic regression analysis has the advantage of not requiring much rigor in the type or distribution of data. So, in some studies [4-12], FIM scores at discharge or FIM gain are converted to binary data of 0 and 1 , and used in logistic regression analysis. In these studies, FIM scores at discharge or FIM gain was set to 1 (favorable outcome) if the patient's score was equal to or above a fixed score, and 0 (unfavorable outcome) if the score was less than the fixed score. Specifically, FIM scores at discharge of 80 points or above [4-6], and the median value or above of motor FIM gain [7-12], were set as favorable outcomes.

However, raising the FIM score at discharge to 80 points is more difficult for patients having FIM scores at admission of 18 points than for those with scores of 79 points. As for FIM gain, because the relationship between motor FIM score at admission and motor FIM gain shows a bell curve [13], the difficulty of motor FIM gain to exceed the median value is different between patients having motor
FIM scores at admission of 13 points (total assistance level) and those having a score of 40 points (moderate assistance level). In other words, in conventional methods [4-12], whether or not FIM score at discharge or FIM gain becomes greater than the fixed score depends on the FIM scoresatadmission, ratherthanthefactorssuchastheamountoftraining.

In this study, a logistic regression analysis was performed on data of patients with stroke hospitalized in a convalescent rehabilitation ward in Japan [14] with FIM at discharge (0 or 1) as the objective variable. We compared the following two methods: a conventional method that sets FIM scores at discharge of 80 points or above as a favorable outcome and a new method that stratifies FIM scores at admission into seven groups, and the median value or above of FIM scores at discharge in each group is set as a favorable outcome. The purpose of this study was to show that the new method can evaluate the factors influencing FIM improvement without being affected by FIM scores at admission.

"Corresponding Author: Dr. Makoto Tokunaga, Department of Rehabilitation, Kumamoto Kinoh Hospital, 6-8-1 Yamamuro, Kita-ku, Kumamoto 860-8518, Japan, Tel: +8196345 8111; E-mail: tokunaga@juryo.or.jp

Citation: Tokunaga M, Tanaka H, Nishimura N, Sugihara K, Kamoshita H (2018) The Threshold for Favorable Outcome should be Set According to Functional Independence Measure Score at Admission in Logistic Regression Analysis. Int J Phys Ther Rehab 4: 139. doi: https://doi.org/10.15344/2455-7498/2017/139

Copyright: (C) 2018 Tokunaga et al. This is an open-access article distributed under the terms of the Creative Commons Attribution License, which permits unrestricted use, distribution, and reproduction in any medium, provided the original author and source are credited. 
Citation: Tokunaga M, Tanaka H, Nishimura N, Sugihara K, Kamoshita H (2018) The Threshold for Favorable Outcome should be Set According to Functional Independence Measure Score at Admission in Logistic Regression Analysis. Int J Phys Ther Rehab 4: 139. doi: https://doi.org/10.15344/2455-7498/2017/139

Page 2 of 4

\section{Methods}

\section{Patients}

A total of 770 stroke patients who were admitted to the convalescent rehabilitation ward in A Hospital between April 1, 2013 and June 17, 2016, after undergoing treatment at acute phase hospitals, were enrolled. The following patients were excluded: those with subarachnoid hemorrhage, those aged younger than 40 years old, those who died in the hospital, those whose outcome was not recorded, those admitted within 6 days or more than 61 days after onset, those who spent less than 31 days in the hospital, those whose FIM score at admission or discharge was not recorded, those who were readmitted, those whose motor FIM score at admission was 91 points, and those whose motor FIM gain was less than 0 pint, those whose FIM score at admission was 80 points and above. The remaining 290 patients were included in this study (Table 1).

\begin{tabular}{|l|l|}
\hline Number of patients & 290 \\
\hline Age & $72.6 \pm 11.6(74)$ \\
\hline Sex & Male 166, female 124 \\
\hline Infarction, hemorrhage & 161,129 \\
\hline Number of days from onset to admission & $18.4 \pm 9.5(16)$ \\
\hline Number of days in hospital & $109.3 \pm 30.6(112)$ \\
\hline Motor FIM score at admission & $28.0 \pm 14.1(25)$ \\
\hline Cognitive FIM score at admission & $17.4 \pm 8.2(17)$ \\
\hline Total FIM score at admission & $45.4 \pm 20.0(44)$ \\
\hline Motor FIM score at discharge & $53.1 \pm 23.8(56.5)$ \\
\hline Cognitive FIM score at discharge & $22.6 \pm 8.7(24)$ \\
\hline Total FIM score at discharge & $75.7 \pm 31.2(80)$ \\
\hline Motor FIM gain & $25.1 \pm 16.8(25)$ \\
\hline Cognitive FIM gain & $5.2 \pm 5.2(4)$ \\
\hline Total FIM gain & $30.3 \pm 19.7(30)$ \\
\hline $\begin{array}{l}\text { Table 1: Clinical characteristics of subjects in this study. } \\
\text { FIM, Functional Independence Measure. } \\
\text { Data for this study are expressed as } \\
\text { mean } \pm \text { ntandard deviation (median value) }\end{array}$ \\
\hline
\end{tabular}

Conventional method that sets FIM scores at discharge of 80 points or above as a favorable outcome.

Logistic regression analysis was performed on six explanatory variables including age, sex, type of stroke (brain infarction or brain hemorrhage), number of days from onset to admission, motor FIM score at admission, and cognitive FIM score at admission. These six explanatory variables were chosen because they were often used in multiple regression analyses $[1,2]$. The objective variable was FIM at discharge (0 or 1). Similar to the previous studies [4-6], FIM scores at discharge of 80 points or above were set as 1 (favorable outcome) and those less than 80 points were set as 0 (unfavorable outcome).

New method that stratifies FIM scores at admission into seven groups, and the median value or above of FIM scores at discharge in each group is set as a favorable outcome.

First, FIM scores at admission were stratified into seven groups: i.e. $18-19$ points, $20-29$ points, $30-39$ points, $40-49$ points, $50-59$ points, 60-69 points, and 70-79 points. The median value of FIM scores at discharge in each group were 21 points, 48.5 points, 68.5 points, 68 points, 103 points, 103.5 points, and 106 points, respectively. The median value or above was set as 1 (favorable outcome) and a score less than the median value was set as 0 (unfavorable outcome) in each group. So, there were seven thresholds according to FIM scores at admission (Figure 1). Logistic regression analysis was performed using the same explanatory variables as the conventional method.

This study complied with the regulations of the Clinical Research Ethics Committee of the first author's hospital. All personal data were processed so as not to identify any individuals. The statistics software we used was IBM SPSS Statistics version 24.

\section{Results}

In the conventional method, the higher the FIM scores at admission, the greater the proportion of patients who had an FIM at discharge of 1 ( $\geq 80$ points) (Figure 2 ). In logistic regression analysis, both motor FIM score at admission and cognitive FIM score at admission were significant explanatory variables (Table 2a).

In the new method, the proportions of patients who had favorable outcome were about $50 \%$ in all seven groups stratified by FIM scores at admission. In logistic regression analysis, neither motor FIM score at admission nor cognitive FIM score at admission was a significant explanatory variable (Table $2 \mathrm{~b}$ ).

\section{Discussion}

We herein reported a new method of setting FIM at discharge (objective variable) as 1 (favorable outcome) according to FIM scores at admission in logistic regression analysis.

Both motor FIM score at admission and cognitive FIM score at admission were significant explanatory variables, and their odds ratios were greater than 1.0 in the conventional method. This indicates that if FIM score at admission is higher, FIM score at discharge is likely to be 80 points or above. This was also shown in Figure 2. These results indicate that whether favorable outcome or not is dependent on FIM scores at admission.

On the other hand, neither motor FIM score at admission nor cognitive FIM score at admission was a significant explanatory variable in the new method. The proportion of patients who had favorable outcome was almost the same among seven groups stratified by FIM scores at admission. These results indicate that the new method is less susceptible to FIM scores at admission. So, this new method is thought to be able to evaluate the factors influencing FIM improvement without being affected by FIM scores at admission.

Regarding the logistic regression analysis in which the median value or above of FIM gain is set to 1 (favorable outcome) [7-12], stratification by FIM scores at admission should also be necessary. And there are also some studies that set the Barthel index score at discharge of 60 points or above and a modified Rankin Scale score of 2 or less at discharge as favorable outcomes [15]. The difficulty to achieve a Barthel index score at discharge of 60 points is different between patients having Barthel index scores of 0 point at admission and those having 55 points. Therefore, stratification of the Barthel index score at admission should be necessary. 
Citation: Tokunaga M, Tanaka H, Nishimura N, Sugihara K, Kamoshita H (2018) The Threshold for Favorable Outcome should be Set According to Functional Independence Measure Score at Admission in Logistic Regression Analysis. Int J Phys Ther Rehab 4: 139. doi: https://doi.org/10.15344/2455-7498/2017/139

Page 3 of 4

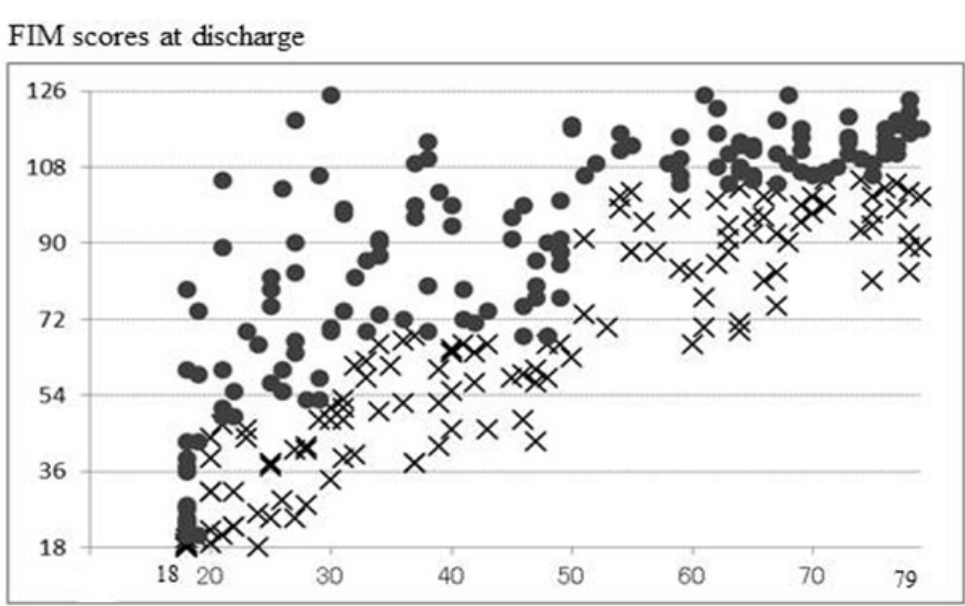

FIM scores at admission

Figure 1: Patient distribution. •, Patients who had FIM at discharge of 1; X, Patients who had FIM at discharge of 0.

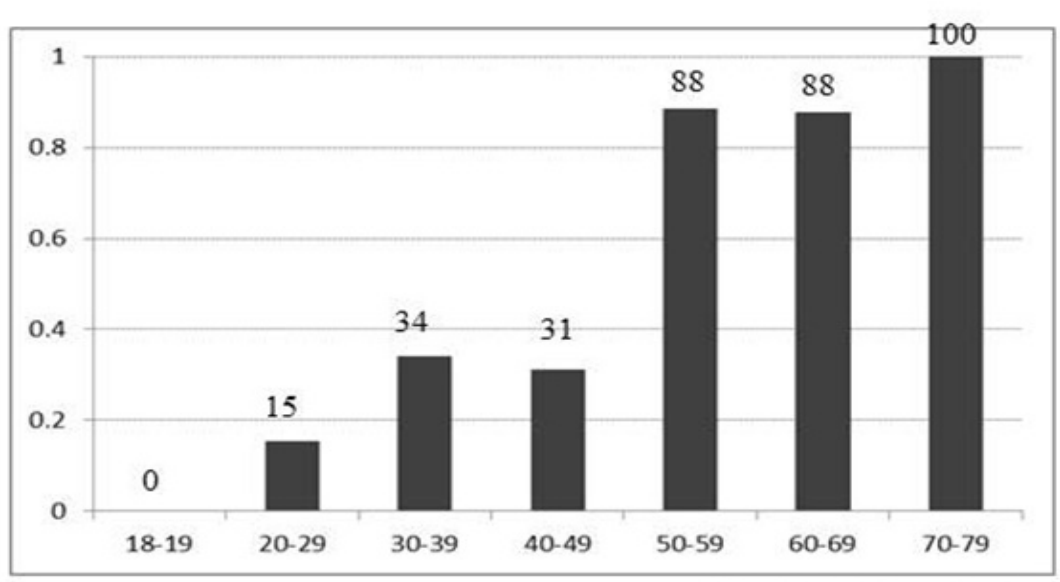

FIM scores at admission

Figure 2: Proportion of patients who had favorable outcome. Numerical value, percentage of patients who had favorable outcome.

\begin{tabular}{|l|c|c|c|}
\hline \multicolumn{2}{|l|}{ a) Conventional method } & OR & p5\%CI \\
\hline Age & 0.881 & $0.846-0.932$ & 0.000 \\
\hline Male 0, female 1 & 0.396 & $0.171-0.915$ & 0.030 \\
\hline Infarction 0, hemorrhage 1 & 1.603 & $0.639-4.019$ & 0.315 \\
\hline Number of days from onset to admission & 0.923 & $0.880-0.968$ & 0.001 \\
\hline Motor FIM score at admission & 1.134 & $1.089-1.181$ & 0.000 \\
\hline Cognitive FIM score at admission & 1.204 & $1.122-1.293$ & 0.000 \\
\hline b) New method & & & 0.000 \\
\hline & OR & $0.85 \%$ CI \\
\hline Age & 0.908 & $0.291-0.856$ & 0.012 \\
\hline Male 0, female 1 & 0.499 & $0.462-1.523$ & 0.001 \\
\hline Infarction 0, hemorrhage 1 & 0.838 & $0.914-0.976$ & 0.149 \\
\hline Number of days from onset to admission & 0.944 & $0.960-1.006$ & 0.588 \\
\hline Motor FIM score at admission & 0.983 & $0.950-1.029$ & \\
\hline Cognitive FIM score at admission & 0.989 & & 0.000 \\
\hline
\end{tabular}

Table 2: Logistic regression analysis.

OR, Odds ratio; CI, Confidence interval; p, p value; Objective variable, FIM at discharge (0 or 1). 
Citation: Tokunaga M, Tanaka H, Nishimura N, Sugihara K, Kamoshita H (2018) The Threshold for Favorable Outcome should be Set According to Functional Independence Measure Score at Admission in Logistic Regression Analysis. Int J Phys Ther Rehab 4: 139. doi: https://doi.org/10.15344/2455-7498/2017/139

Page 4 of 4

There are some limitations in this study. First, it can't be asserted that the six explanatory variables used are most appropriate in logistic regression analysis. Second, we can't definitely say that the odds ratios in the new method are more accurate than those in the conventional method. Third, multiple regression analysis is more commonly used in studies to find out how much influence the factor has on FIM outcome than the logistic regression analysis. Which method, multiple regression analysis or logistic regression analysis, is more appropriate for these studies is not known. The predictive accuracy of multiple regression analysis is not satisfactory [3]. Logistic regression analysis has the advantage of not requiring much rigor in the type or distribution of data, but has the disadvantage of losing a lot of information in the process of converting quantitative data of FIM into binary data of 0 and 1 .

\section{Conclusion}

The conventional logistic regression analysis that sets one fixed value (FIM score at discharge $\geq 80$ points) as favorable outcome has a problem that it is heavily influenced by FIM scores at admission. In studies evaluating the effect of factors influencing FIM improvement, the thresholds for favorable outcome should be set according to FIM scores at admission.

\section{Competing Interests}

No benefits in any form have been or will be received from any commercial party related directly or indirectly to the subject of this manuscript.

\section{Author Contributions}

Makoto Tokunaga analyzed clinical data and drafted the manuscript. Hirotaka Tanaka, Naoji Nishimura, Katsunobu Sugihara, and Hiroshi Kamoshita gave important suggestions. All authors read and approved the manuscript.

\section{References}

1. Meyer MJ, Pereira S, McClure A, Teasell R, Thind A, et al. (2015) A systematic review of studies reporting multivariable models to predict functional outcomes after post-stroke inpatient rehabilitation. Disabil Rehabil 37: 1316-1323.

2. Tokunaga M, Hori K, Ehara K, Murao S, Akase R, et al. (2016) Review of multiple regression analysis for stroke patients hospitalized in Kaifukuki rehabilitation ward. J Clin Rehabil 25: 821-826.

3. Tokunaga $M$, Hashimoto $Y$, Watanabe $S$, Nakanishi $R$, Yamanaga $H$, et al. (2017) Methods for Improving the Predictive accuracy using multiple linear regression analysis to predict the improvement degree of Functional Independence Measure for stroke patients. Int J Phys Med Rehabili 5: 414.

4. Mizrahi EH, Fleissig Y, Arad M, Adunsky A (2005) Plasma homocysteine level and functional outcome of patients with ischemic stroke. Arch Phys Med Rehabil 86: 60-63.

5. Adunsky A, Arad M, Blumstein T, Weitzman A, Mizrahi EH, et al. (2008) Discharge hemoglobin and functional outcome of elderly hip fractured patients undergoing rehabilitation. Eur J Phys Rehabil Med 44: 417-422.

6. Mizrahi EH, Arad M, Adunsky A (2016) Pre-stroke dementia does not affect the post-acute care functional outcome of old patients with ischemic stroke. Geriatr Gerontol Int 16: 928-933.

7. Mizrahi EH, Fleissig Y, Arad M, Adunski A (2006) Functional outcome of elderly hip fracture patients, does diabetes matter? Arch Geront Geriatr 43: 165-173.
8. Tokunaga M, Beppu A, Tamura Y, Oowaki K, Tokunaga $Y$, et al. (2016) Relationship between improvement in GNRI, a nutritional index, and improvement in motor FIM in elderly stroke patients hospitalized in a Kaifukuki Rehabilitation Ward. Jpn J Compr Rehabil Sci 7: 7-12.

9. Tang V , Harvey D, Park Dorsay J, Jiang S, Rathbone MP, et al. (2007) Prognostic indicators in metastatic spinal cord compression, using functional independence measure and Tokuhashi scale to optimize rehabilitation planning. Spinal Cord 45: 671-677.

10. Naruishi K, Kunita A, Kubo K, Nagata T, Takashiba S, et al. (2014) Predictors of improved functional outcome in elderly inpatients after rehabilitation, a retrospective study. Clin Interv Aging 9: 2133-2141.

11. Gotou R, Tanaka N, Watanabe H, Kanamori T, Yanagi H, et al. (2014) Factors influencing the recovery of the ability to perform activities of daily living of inpatients with disuse syndrome. Rigaku-ryohou Kagaku 29: 751-758.

12. Matsuo H, Sonoda S, Maeshima S, Watanabe M, Sasaki S, et al. (2016) Contribution of physical impairment or imaging findings in the prediction of $\mathrm{ADL}$ outcome in stroke patients with middle cerebral artery infarction. Jpn J Compr Rehabil Sci 7: 119-129.

13. Tokunaga M, Mita S, Tashiro K, Yamaga M, Hashimoto $Y$, et al. (2017) Methods for comparing Functional Independence Measure (FIM) improvement degree for stroke patients between rehabilitation hospitals. Int J Phys Med Rehabil 5: 394.

14. Miyai I, Sonoda S, Nagai S, Takayama Y, Inoue Y, et al. (2011) Results of new policies for inpatient rehabilitation coverage in Japan. Neurorehabil Neural Repair 25: 540-547.

15. Doehner W, Schenkel J, Anker SD, Springer J, Audebert HJ, et al. (2013) Overweight and obesity are associated with improved survival, functional outcome, and stroke recurrence after acute stroke or transient ischaemic attack. Eur Heart J 34: 268-277.
This article was originally published in a special issue: Various Approaches for Rehabilitation Science-Vol II Handled by Editor(s):

Prof. Susumu Ito High-Tech Research Centre Kokushikan Japan 